\title{
Multi-point boundary value problem for first order impulsive integro-differential equations with multi-point jump conditions
}

\author{
Chatthai Thaiprayoon ${ }^{1,3^{*}}$, Decha Samana ${ }^{1,3}$ and Jessada Tariboon ${ }^{2,3}$
}

\author{
* Correspondence: \\ tik_kengjang@hotmail.com \\ 'Department of Mathematics, \\ Faculty of Science, King Mongkut's \\ Institute of Technology \\ Ladkrabang, Bangkok 10520, \\ Thailand \\ Full list of author information is \\ available at the end of the article
}

\begin{abstract}
In this article we introduce a new definition of impulsive conditions for boundary value problems of first order impulsive integro-differential equations with multi-point boundary conditions. By using the method of lower and upper solutions in reversed order coupled with the monotone iterative technique, we obtain the extremal solutions of the boundary value problem. An example is also discussed to illustrate our results,

Mathematics Subject Classification 2010: 34B15; 34B37.

Keywords: impulsive integro-differential equations, multi-point boundary value problem, lower and upper solutions, monotone iterative technique.
\end{abstract}

\section{Introduction}

Impulsive differential equations describe processes which have a sudden change of their state at certain moments. Impulse effects are important in many real world applications, such as physics, medicine, biology, control theory, population dynamics, etc. (see, for example [1-3]). In this article, we consider the following boundary value problem for first order impulsive integro-differential equations (BVP):

$$
\left\{\begin{array}{l}
x^{\prime}(t)=f(t, x(t),(F x)(t),(S x)(t)), \quad t \in J=[0, T], \quad t \neq t_{k} \\
\Delta x\left(t_{k}\right)=I_{k}\left(\sum_{l=1}^{c_{k}} \rho_{l}^{k} x\left(\eta_{l}^{k}\right)\right), \quad k=1,2, \ldots m, \\
x(0)+\mu \sum_{k=1}^{m} \sum_{l=1}^{c_{k}} \tau_{l}^{k} x\left(\eta_{l}^{k}\right)=x(T)
\end{array}\right.
$$

where $f \in C\left(J \times R^{3}, R\right), 0=t_{0}<t_{1}<t_{2}<\cdots<t_{m}<t_{m+1}=T$,

$$
(F x)(t)=\int_{0}^{t} k(t, s) x(s) d s, \quad(S x)(t)=\int_{0}^{T} h(t, s) x(s) d s,
$$

$k \in C\left(D, R^{+}\right), D=\{(t, s) \in J \times J: t \geq s\}, h \in C\left(J \times J, R^{+}\right) . I_{k} \in C(R, R)$, $\Delta x\left(t_{k}\right)=x\left(t_{k}^{+}\right)-x\left(t_{k}^{-}\right), t_{k-1}<\eta_{1}^{k}<\eta_{2}^{k}<\ldots<\eta_{c_{k}}^{k} \leq t_{k}, \tau_{l}^{k}, \rho_{l}^{k} \geq 0, l=1,2, \ldots, c_{k}, c_{k}$ $\in N=\{1,2, \ldots\}, k=1,2, \ldots, m, \mu \geq 0$.

The monotone iterative technique coupled with the method of lower and upper solutions is a powerful method used to approximate solutions of several nonlinear

(c) 2012 Thaiprayoon et al; licensee Springer. This is an Open Access article distributed under the terms of the Creative Commons Attribution License (http://creativecommons.org/licenses/by/2.0), which permits unrestricted use, distribution, and reproduction in any medium, provided the original work is properly cited. 
problems (see [4-14]). Boundary value problems for first order impulsive functional differential equations with lower and upper solutions in reversed order have been widely discussed in recent years (see [15-20]). However, the discussion of multi-point boundary value problems for first order impulsive functional differential equations is very limited (see [21]). In all articles concerned with applications of the monotone iterative technique to impulsive problems, the authors have assumed that $\Delta x\left(t_{k}\right)=I_{k}\left(x\left(t_{k}\right)\right)$, that is a short-term rapid change of the state at impulse point $t_{k}$ depends on the left side of the limit of $x\left(t_{k}\right)$.

Recently, Tariboon [22] and Liu et al. [23] studied some types of impulsive boundary value problems with the impulsive integral conditions

$$
\Delta x\left(t_{k}\right)=I_{k}\left(\int_{t_{k}-\tau_{k}}^{t_{k}} x(s) d s-\int_{t_{k-1}}^{t_{k-1}+\sigma_{k-1}} x(s) d s\right), \quad k=1,2, \ldots, m .
$$

It should be noticed that the terms $\int_{t_{k}-\tau_{k}}^{t_{k}} x(s) d s$ and $\int_{t_{k-1}}^{t_{k-1}+\sigma_{k-1}} x(s) d s$ of impulsive condition (1.2) illustrate the past memory state on $\left[t_{k}-\tau_{k}, t_{k}\right]$ before impulse points $t_{k}$ and the history effects after the past impulse points $t_{k-1}$ on $\left(t_{k-1}, t_{k-1}+\sigma_{k-1}\right]$, respectively.

The aim of the present article is to discuss the new impulsive multi-point condition

$$
\Delta x\left(t_{k}\right)=I_{k}\left(\sum_{l=1}^{c_{k}} \rho_{l}^{k} x\left(\eta_{l}^{k}\right)\right)=I_{k}\left(\rho_{1}^{k} x\left(\eta_{1}^{k}\right)+\cdots+\rho_{l}^{k} x\left(\eta_{l}^{k}\right)+\cdots+\rho_{c_{k}}^{k} x\left(\eta_{c_{k}}^{k}\right)\right),
$$

for $t_{k-1}<\eta_{1}^{k}<\eta_{2}^{k}<\cdots<\eta_{c_{k}}^{k} \leq t_{k}, k=1,2, \ldots, m$. The new jump conditions mean that a sudden change of the state at impulse point $t_{k}$ depends on the multi-point $\eta_{l}^{k}\left(l=1,2, \ldots, c_{k}\right)$ of past states on $\left(t_{k-1}, t_{k}\right]$. We note that if $c_{k}=1, \eta_{c_{k}}^{k}=t_{k}$ and $\rho_{c_{k}}^{k}=1$, then the impulsive condition (1.3) is reduced to the simple impulsive condition $\Delta x\left(t_{k}\right)=I_{k}$ $\left(x\left(t_{k}\right)\right)$.

Firstly, we introduce the definitions of lower and upper solutions and formulate some lemmas which are used in our discussion. In the main results, we obtain the existence of extreme solutions for BVP (1.1) by using the method of lower and upper solutions in reversed order and the monotone iterative technique. Finally, we give an example to illustrate the obtained results.

\section{Preliminaries}

Let $J=J \backslash\left\{t_{1}, t_{2}, \ldots, t_{m}\right\} . P C(J, R)=\{x: J \rightarrow R ; x(t)$ is continuous everywhere except for some $t_{k}$ at which $x\left(t_{k}^{-}\right)$and $x\left(t_{k}^{+}\right)$exist and $\left.x\left(t_{k}^{-}\right)=x\left(t_{k}\right), k=1,2, \ldots, m\right\}, P C^{1}(J, R)=$ $\left\{x \in P C(J, R) ; x^{\prime}(t)\right.$ is continuous everywhere except for some $t_{k}$ at which $x^{\prime}\left(t_{k}^{+}\right)$and $x^{\prime}\left(t_{k}^{-}\right)$exist and $\left.x^{\prime}\left(t_{k}^{-}\right)=x^{\prime}\left(t_{k}\right)\right\}$. Let $E=P C(J, R)$ and $\mathcal{F}=P C^{1}(J, R)$, then $E$ and $\mathcal{F}$ are Banach spaces with the nomes $\|x\|_{E}=\sup _{t \in J}|x(t)|$ and $\|x\|_{\mathcal{F}}=\max \left\{\|x\|_{E^{\prime}}\left\|x^{\prime}\right\|_{E}\right\}$, respectively. A function $x \in \mathcal{F}$ is called a solution of BVP (1.1) if it satisfies (1.1).

Definition 2.1. A function $\alpha_{0} \in \mathcal{F}$ is called a lower solution of BVP (1.1) if:

$$
\left\{\begin{array}{l}
\alpha^{\prime}{ }_{0}(t) \leq f\left(t, \alpha_{0}(t),\left(F \alpha_{0}\right)(t),\left(S \alpha_{0}\right)(t)\right), \quad t \in J^{-}, \\
\Delta \alpha_{0}\left(t_{k}\right) \leq I_{k}\left(\sum_{l=1}^{c_{k}} \rho_{l}^{k} \alpha_{0}\left(\eta_{l}^{k}\right)\right), \quad k=1,2, \ldots, m, \\
\alpha_{0}(0)+\mu \sum_{k=1}^{m} \sum_{l=1}^{c_{k}} \tau_{l}^{k} \alpha_{0}\left(\eta_{l}^{k}\right) \leq \alpha_{0}(T) .
\end{array}\right.
$$


Analogously, a function $\beta_{0} \in \mathcal{F}$ is called an upper solution of BVP (1.1) if:

$$
\left\{\begin{array}{l}
\beta_{0}^{\prime}(t) \geq f\left(t, \beta_{0}(t),\left(F \beta_{0}\right)(t),\left(S \beta_{0}\right)(t)\right), \quad t \in J^{-}, \\
\Delta \beta_{0}\left(t_{k}\right) \geq I_{k}\left(\sum_{l=1}^{c_{k}} \rho_{l}^{k} \beta_{0}\left(\eta_{l}^{k}\right)\right), \quad k=1,2, \ldots, m \\
\beta_{0}(0)+\mu \sum_{k=1}^{m} \sum_{l=1}^{c_{k}} \tau_{l}^{k} \beta_{0}\left(\eta_{l}^{k}\right) \geq \beta_{0}(T),
\end{array}\right.
$$

where $t_{k-1}<\eta_{l}^{k} \leq t_{k}, \rho_{l}^{k}, \tau_{l}^{k} \geq 0, l=1,2, \ldots, c_{k} c_{k} \in N=\{1,2, \ldots\}, k=1,2, \ldots, m$ and $\mu \geq 0$.

Let us consider the following boundary value problem of a linear impulsive integrodifferential equation (BVP):

$$
\left\{\begin{array}{l}
x^{\prime}(t)-M x(t)=H(F x)(t)+K(S x)(t)+v(t), \quad t \in J^{-}, \\
\Delta x\left(t_{k}\right)=L_{k} \sum_{l=1}^{c_{k}} \rho_{l}^{k} x\left(\eta_{l}^{k}\right)+I_{k}\left(\sum_{l=1}^{c_{k}} \rho_{l}^{k} \sigma\left(\eta_{l}^{k}\right)\right) \\
\quad-L_{k} \sum_{l=1}^{c_{k}} \rho_{l}^{k} \sigma\left(\eta_{l}^{k}\right), \quad k=1,2, \ldots, m, \\
x(0)+\mu \sum_{k=1}^{m} \sum_{l=1}^{c_{k}} \tau_{l}^{k} \sigma\left(\eta_{l}^{k}\right)=x(T),
\end{array}\right.
$$

where $M>0, H, K \geq 0, L_{k} \geq 0, t_{k-1}<\eta_{l}^{k} \leq t_{k}, \tau_{l}^{k}, \rho_{l}^{k} \geq 0, l=1,2, \ldots, c_{k}, c_{k} \in N=\{1$, $2, \ldots\}, k=1,2, \ldots, m$ are constants and $v(t), \sigma(t) \in E$.

Lemma 2.1. $x \in \mathcal{F}$ is a solution of (2.1) if and only if $x \in E$ is a solution of the impulsive integral equation

$$
\begin{aligned}
x(t)= & \frac{\mu e^{M t}}{e^{M T}-1} \sum_{k=1}^{m} \sum_{l=1}^{c_{k}} \tau_{l}^{k} \sigma\left(\eta_{l}^{k}\right)-\int_{0}^{T} G(t, s) P(s) d s-\sum_{k=1}^{m} G\left(t, t_{k}\right)\left[L_{k} \sum_{l=1}^{c_{k}} \rho_{l}^{k} x\left(\eta_{l}^{k}\right)\right. \\
& \left.+I_{k}\left(\sum_{l=1}^{c_{k}} \rho_{l}^{k} \sigma\left(\eta_{l}^{k}\right)\right)-L_{k} \sum_{l=1}^{c_{k}} \rho_{l}^{k} \sigma\left(\eta_{l}^{k}\right)\right], \quad t \in J,
\end{aligned}
$$

where $P(t)=H(F x)(t)+K(S x)(t)+v(t)$ and

$$
G(t, s)=\left\{\begin{array}{l}
\frac{e^{M(t-s)}}{e^{M T}-1}, 0 \leq s<t \leq T \\
\frac{e^{M(T+t-s)}}{e^{M T}-1}, 0 \leq t \leq s \leq T
\end{array}\right.
$$

Proof. Assume that $x(t)$ is a solution of BVP (2.1). By using the variation of parameters formula, we get

$$
\begin{aligned}
x(t)= & x(0) e^{M t}+\int_{0}^{t} e^{M(t-s)} P(s) d s+\sum_{0<t_{k}<t} e^{M\left(t-t_{k}\right)}\left[L_{k} \sum_{l=1}^{c_{k}} \rho_{l}^{k} x\left(\eta_{l}^{k}\right)+I_{k}\left(\sum_{l=1}^{c_{k}} \rho_{l}^{k} \sigma\left(\eta_{l}^{k}\right)\right)\right. \\
& \left.-L_{k} \sum_{l=1}^{c_{k}} \rho_{l}^{k} \sigma\left(\eta_{l}^{k}\right)\right] .
\end{aligned}
$$

Putting $t=T$ in (2.3), we have

$$
\begin{aligned}
x(T)= & x(0) e^{M T}+\int_{0}^{T} e^{M(T-s)} P(s) d s+\sum_{k=1}^{m} e^{M\left(T-t_{k}\right)}\left[L_{k} \sum_{l=1}^{c_{k}} \rho_{l}^{k} x\left(\eta_{l}^{k}\right)+I_{k}\left(\sum_{l=1}^{c_{k}} \rho_{l}^{k} \sigma\left(\eta_{l}^{k}\right)\right)\right. \\
& \left.-L_{k} \sum_{l=1}^{c_{k}} \rho_{l}^{k} \sigma\left(\eta_{l}^{k}\right)\right] .
\end{aligned}
$$


From $x(0)+\mu \sum_{k=1}^{m} \sum_{l=1}^{c_{k}} \tau_{l}^{k} \sigma\left(\eta_{l}^{k}\right)=x(T)$, we obtain

$$
\begin{aligned}
x(0)= & \frac{-1}{e^{M T}-1}\left\{-\mu \sum_{k=1}^{m} \sum_{l=1}^{c_{k}} \tau_{l}^{k} \sigma\left(\eta_{l}^{k}\right)+\int_{0}^{T} e^{M(T-s)} P(s) d s\right. \\
& \left.+\sum_{k=1}^{m} e^{M\left(T-t_{k}\right)}\left[L_{k} \sum_{l=1}^{c_{k}} \rho_{l}^{k} x\left(\eta_{l}^{k}\right)+I_{k}\left(\sum_{l=1}^{c_{k}} \rho_{l}^{k} \sigma\left(\eta_{l}^{k}\right)\right)-L_{k} \sum_{l=1}^{c_{k}} \rho_{l}^{k} \sigma\left(\eta_{l}^{k}\right)\right]\right\} .
\end{aligned}
$$

Substituting (2.5) into (2.3), we see that $x \in E$ satisfies (2.2). Hence, $x(t)$ is also the solution of (2.2).

Conversely, we assume that $x(t)$ is a solution of (2.2). By computing directly, we have

$$
G_{t}^{\prime}(t, s)=\left\{\begin{array}{ll}
\frac{M e^{M(t-s)}}{e^{M T}-1}, & 0 \leq s<t \leq T, \\
\frac{M e^{M(T+t-s)}}{e^{M T}-1}, & 0 \leq t \leq s \leq T
\end{array}=M G(t, s)\right.
$$

Differentiating (2.2) for $t \neq t_{k}$, we obtain

$$
x^{\prime}(t)=M x(t)+H(F x)(t)+K(S x)(t)+v(t) .
$$

It is easy to see that

$$
\Delta x\left(t_{k}\right)=L_{k} \sum_{l=1}^{c_{k}} \rho_{l}^{k} x\left(\eta_{l}^{k}\right)+I_{k}\left(\sum_{l=1}^{c_{k}} \rho_{l}^{k} \sigma\left(\eta_{l}^{k}\right)\right)-L_{k} \sum_{l=1}^{c_{k}} \rho_{l}^{k} \sigma\left(\eta_{l}^{k}\right)
$$

Since $G(0, s)=G(T, s)$, then $x(0)+\mu \sum_{k=1}^{m} \sum_{l=1}^{c_{k}} \tau_{l}^{k} \sigma\left(\eta_{l}^{k}\right)=x(T)$. This completes the proof. $\square$

Lemma 2.2. Assume that $M>0, H, K \geq 0, L_{k} \geq 0, t_{k-1}<\eta_{l}^{k} \leq t_{k}, \rho_{l}^{k} \geq 0, l=1,2, \ldots$, $c_{k}, c_{k} \in N=\{1,2, \ldots\}, k=1,2, \ldots, m$, and the following inequality holds:

$$
\frac{e^{M T}}{e^{M T}-1} \int_{0}^{T}\left[H \int_{0}^{s} k(s, r) d r+K \int_{0}^{T} h(s, r) d r\right] d s+\frac{e^{M T}}{e^{M T}-1} \sum_{k=1}^{m} L_{k}\left(\sum_{l=1}^{c_{k}} \rho_{l}^{k}\right)<1
$$

Then BVP (2.1) has a unique solution.

Proof. For any $x \in E$, we define an operator $A$ by

$$
\begin{aligned}
(A x)(t)= & \frac{\mu e^{M} t}{e^{M T}-1} \sum_{k=1}^{m} \sum_{l=1}^{c_{k}} \tau_{l}^{k} \sigma\left(\eta_{l}^{k}\right)-\int_{0}^{T} G(t, s)[H(F x)(s)+K(S x)(s)+v(s)] d s \\
& -\sum_{k=1}^{m} G\left(t, t_{k}\right)\left[L_{k} \sum_{l=1}^{c_{k}} \rho_{l}^{k} x\left(\eta_{l}^{k}\right)+I_{k}\left(\sum_{l=1}^{c_{k}} \rho_{l}^{k} \sigma\left(\eta_{l}^{k}\right)\right)-L_{k} \sum_{l=1}^{c_{k}} \rho_{l}^{k} \sigma\left(\eta_{l}^{k}\right)\right], \quad t \in J,
\end{aligned}
$$

where $G(t, s)$ is defined as in Lemma 2.1. Since $\max _{t \in[0, T]}\{G(t, s)\}=\frac{e^{M T}}{e^{M T}-1}$, we have for any $x, y \in E$, that 


$$
\begin{aligned}
\|A x-A y\|_{E}= & -\int_{0}^{T} G(t, s)\left[H \int_{0}^{s} k(s, r)(x(r)-y(r)) d r\right. \\
& \left.+K \int_{0}^{T} h(s, r)(x(r)-\gamma(r)) d r\right] d s \\
& -\sum_{k=1}^{m} G\left(t, t_{k}\right) L_{k} \sum_{l=1}^{c_{k}} \rho_{l}^{k}\left(x\left(\eta_{l}^{k}\right)-\gamma\left(\eta_{l}^{k}\right)\right) \mid \\
\leq & {\left[\frac{e^{\mathrm{MT}}}{e^{\mathrm{MT}}-1} \int_{0}^{T}\left[H \int_{0}^{s} k(s, r) d r+K \int_{0}^{T} h(s, r) d r\right] d s\right.} \\
& \left.+\frac{e^{\mathrm{MT}}}{e^{\mathrm{MT}}-1} \sum_{k=1}^{m} L_{k}\left(\sum_{l=1}^{c_{k}} \rho_{l}^{k}\right)\right]\|x-\gamma\|_{E} .
\end{aligned}
$$

From (2.6) and the Banach fixed point theorem, $A$ has a unique fixed point $\bar{x} \in E$. By Lemma 2.1, $\bar{x}$ is also the unique solution of (2.1).

Lemma 2.3. Assume that $x \in \mathcal{F}$ satisfies

$$
\left\{\begin{array}{l}
x^{\prime}(t) \geq M x(t)+H(F x)(t)+K(S x)(t), \quad t \in J^{-}, \\
\Delta x\left(t_{k}\right) \geq L_{k} \sum_{l=1}^{c_{k}} \rho_{l}^{k} x\left(\eta_{l}^{k}\right), \quad k=1,2, \ldots, m, \\
x(0) \geq x(T),
\end{array}\right.
$$

where $M>0, H, K \geq 0, L_{k} \geq 0, t_{k-1}<\eta_{l}^{k} \leq t_{k}, \rho_{l}^{k} \geq 0, l=1,2, \ldots, c_{k}, c_{k} \in N=\{1,2$, ...\}, $k=1,2, \ldots, m$. In addition assume that

$$
e^{\mathrm{MT}}\left[\int_{0}^{T} q(s) d s+\sum_{k=1}^{m} L_{k} \sum_{l=1}^{c_{k}} \rho_{l}^{k} e^{-M\left(t_{k}-\eta_{l}^{k}\right)}\right] \leq 1,
$$

where $q(t)=H \int_{0}^{t} k(t, s) e^{-M(t-s)} d s+K \int_{0}^{T} h(t, s) e^{-M(t-s)} d s$. Then, $x(t) \leq 0$ for all $t \in$ $J$.

Proof. Set $u(t)=x(t) e^{-M t}$ for $t \in J$, then we have

$$
\left\{\begin{array}{l}
u^{\prime}(t) \geq H \int_{0}^{t} k(t, s) e^{-M(t-s)} u(s) d s+K \int_{0}^{T} h(t, s) e^{-M(t-s)} u(s) d s, \quad t \in J^{-}, \\
\Delta u\left(t_{k}\right) \geq L_{k} \sum_{l=1}^{c_{k}} \rho_{l}^{k} e^{-M\left(t_{k}-\eta_{l}^{k}\right)} u\left(\eta_{l}^{k}\right), \quad k=1,2, \ldots, m, \\
u(0) \geq e^{M T} u(T) .
\end{array}\right.
$$

Obviously, the function $u(t)$ and $x(t)$ have the same sign. Suppose, to the contrary, that $u(t)>0$ for some $t \in J$. Then, there are two cases:

(i) There exists a $t^{*} \in J$, such that $u\left(t^{*}\right)>0$ and $u(t) \geq 0$ for all $t \in J$.

(ii) There exists $t^{*}, t_{*} \in J$, such that $u\left(t^{*}\right)>0$ and $u\left(t_{*}\right)<0$.

Case (i): Equation (2.10) implies that $u^{\prime}(t) \geq 0$ for $t \in J$ and $\Delta u\left(t_{k}\right) \geq 0$ for $k=1,2$, ..., $m$. This means that $u(t)$ is nondecreasing in $J$. Therefore, $u(T) \geq u\left(t^{*}\right)>0$ and $u(T) \geq$ $u(0) \geq u(T) e^{M T}$, which is a contradiction.

Case (ii): Let $t_{*} \in\left(t_{i} t_{i+1}\right], i \in\{0,1, \ldots, m\}$, such that $u\left(t_{m}\right)=\inf \left\{u(t): t \in \mathcal{J}<0\right.$ and $t^{*}$ $\in\left(t_{j}, t_{j+1}\right], j \in\{0,1, \ldots, m\}$, such that $u\left(t^{*}\right)>0$. We first claim that $u(0) \leq 0$. Otherwise, if $u(0)>0$, then by (2.10), we have 


$$
\begin{aligned}
u\left(t_{*}\right)-u(0) \geq & H \int_{0}^{t_{*}} \int_{0}^{s} k(s, r) e^{-M(s-r)} u(r) d r d s \\
& +K \int_{0}^{t_{*}} \int_{0}^{T} h(s, r) e^{-M(s-r)} u(r) d r d s+\sum_{k=1}^{i} \Delta u\left(t_{k}\right) \\
\geq & u\left(t_{*}\right)\left[\int_{0}^{t_{*}} q(s) d s+\sum_{k=1}^{i} L_{k} \sum_{l=1}^{c_{k}} \rho_{l}^{k} e^{-M\left(t_{k}-\eta_{l}^{k}\right)}\right] \\
\geq & u\left(t_{*}\right),
\end{aligned}
$$

a contradiction, and so $u(0) \leq 0$.

If $t^{*}<t_{*}$, then $j \leq i$. Integrating the differential inequality in (2.10) from $t^{*}$ to $t_{*}$, we obtain

$$
\begin{aligned}
u\left(t_{*}\right)-u\left(t^{*}\right) \geq & H \int_{t^{*}}^{t_{*}} \int_{0}^{s} k(s, r) e^{-M(s-r)} u(r) d r d s \\
& +K \int_{t^{*}}^{t_{*}} \int_{0}^{T} h(s, r) e^{-M(s-r)} u(r) d r d s+\sum_{k=j+1}^{i} \Delta u\left(t_{k}\right) \\
\geq & u\left(t_{*}\right) \int_{t^{*}}^{t_{*}} q(s) d s+\sum_{k=j+1}^{i} \Delta u\left(t_{k}\right) \\
\geq & u\left(t_{*}\right) \int_{t^{*}}^{t_{*}} q(s) d s+\sum_{k=j+1}^{i} L_{k} \sum_{l=1}^{c_{k}} \rho_{l}^{k} e^{-M\left(t_{k}-\eta_{l}^{k}\right)} u\left(\eta_{l}^{k}\right) \\
\geq & u\left(t_{*}\right)\left[\int_{0}^{T} q(s) d s+\sum_{k=1}^{m} L_{k} \sum_{l=1}^{c_{k}} \rho_{l}^{k} e^{-M\left(t_{k}-\eta_{l}^{k}\right)}\right] \\
\geq & u\left(t_{*}\right),
\end{aligned}
$$

which is a contradiction to $u\left(t^{*}\right)>0$.

Now, assume that $t_{*}<t^{*}$. Since $0 \geq u(0) \geq e^{M T} u(T)$, then $u(T) \leq 0$. From (2.10), we have

$$
\begin{aligned}
u(T)-u\left(t^{*}\right) \geq & H \int_{t^{*}}^{T} \int_{0}^{s} k(s, r) e^{-M(s-r)} u(r) d r d s \\
& +K \int_{t^{*}}^{T} \int_{0}^{T} h(s, r) e^{-M(s-r)} u(r) d r d s+\sum_{k=j+1}^{m} \Delta u\left(t_{k}\right) \\
\geq & u\left(t_{*}\right)\left[\int_{t^{*}}^{T} q(s) d s+\sum_{k=j+1}^{m} L_{k} \sum_{l=1}^{c_{k}} \rho_{l}^{k} e^{-M\left(t_{k}-\eta_{l}^{k}\right)}\right]
\end{aligned}
$$

and $u(0) \geq e^{M T} u(T)$. In consequence,

$$
u(0) \geq e^{M T} u(T) \geq e^{M T} u\left(t^{*}\right)+u\left(t_{*}\right) e^{M T}\left[\int_{t^{*}}^{T} q(s) d s+\sum_{k=j+1}^{m} L_{k} \sum_{l=1}^{c_{k}} \rho_{l}^{k} e^{-M\left(t_{k}-\eta_{l}^{k}\right)}\right]
$$


can be obtained.

If $t_{*}=0$, then

$$
\begin{aligned}
u\left(t_{*}\right) & \geq e^{\mathrm{MT}} u\left(t^{*}\right)+u\left(t_{*}\right) e^{\mathrm{MT}}\left[\int_{t^{*}}^{T} q(s) d s+\sum_{k=j+1}^{m} L_{k} \sum_{l=1}^{c_{k}} \rho_{l}^{k} e^{-M\left(t_{k}-\eta_{l}^{k}\right)}\right] \\
& \geq e^{\mathrm{MT}} u\left(t^{*}\right)+u\left(t_{*}\right) .
\end{aligned}
$$

This contradicts the fact that $u\left(t^{*}\right)>0$.

If $t_{*}>0$, we obtain from (2.11),

$$
u\left(t_{*}\right)-u\left(t_{*}\right)\left[\int_{0}^{t_{*}} q(s) d s+\sum_{k=1}^{i} L_{k} \sum_{l=1}^{c_{k}} \rho_{l}^{k} e^{-M\left(t_{k}-\eta_{l}^{k}\right)}\right] \geq u(0) .
$$

This joint to (2.12) yields

$$
\begin{aligned}
u\left(t_{*}\right)-u\left(t_{*}\right) & {\left[\int_{0}^{t_{*}} q(s) d s+\sum_{k=1}^{i} L_{k} \sum_{l=1}^{c_{k}} \rho_{l}^{k} e^{-M\left(t_{k}-\eta_{l}^{k}\right)}\right] } \\
\geq & e^{\mathrm{MT}} u\left(t^{*}\right)+u\left(t_{*}\right) e^{\mathrm{MT}}\left[\int_{t^{*}}^{T} q(s) d s+\sum_{k=j+1}^{m} L_{k} \sum_{l=1}^{c_{k}} \rho_{l}^{k} e^{-M\left(t_{k}-\eta_{l}^{k}\right)}\right] .
\end{aligned}
$$

Therefore,

$$
\begin{aligned}
u\left(t_{*}\right)-e^{\mathrm{MT}} u\left(t^{*}\right) \geq & u\left(t_{*}\right) e^{\mathrm{MT}}\left[\int_{t^{*}}^{T} q(s) d s+\sum_{k=j+1}^{m} L_{k} \sum_{l=1}^{c_{k}} \rho_{l}^{k} e^{-M\left(t_{k}-\eta_{l}^{k}\right)}\right] \\
& +u\left(t_{*}\right)\left[\int_{0}^{t_{*}} q(s) d s+\sum_{k=1}^{i} L_{k} \sum_{l=1}^{c_{k}} \rho_{l}^{k} e^{-M\left(t_{k}-\eta_{l}^{k}\right)}\right] \\
\geq & u\left(t_{*}\right) e^{\mathrm{MT}}\left[\int_{t^{*}}^{T} q(s) d s+\sum_{k=j+1}^{m} L_{k} \sum_{l=1}^{c_{k}} \rho_{l}^{k} e^{-M\left(t_{k}-\eta_{l}^{k}\right)}\right] \\
& +u\left(t_{*}\right) e^{\mathrm{MT}}\left[\int_{0}^{t_{*}} q(s) d s+\sum_{k=1}^{i} L_{k} \sum_{l=1}^{c_{k}} \rho_{l}^{k} e^{-M\left(t_{k}-\eta_{l}^{k}\right)}\right] \\
\geq & u\left(t_{*}\right) e^{\mathrm{MT}}\left[\int_{0}^{T} q(s) d s+\sum_{k=1}^{m} L_{k} \sum_{l=1}^{c_{k}} \rho_{l}^{k} e^{-M\left(t_{k}-\eta_{l}^{k}\right)}\right] \\
\geq & u\left(t_{*}\right) .
\end{aligned}
$$

This is a contradiction and so $u(t) \leq 0$ for all $t \in J$. The proof is complete.

\section{Main results}

In this section, we are in a position to prove our main results concerning the existence criteria for solutions of BVP (1.1).

For $\beta_{0}, \alpha_{0} \in \mathcal{F}$, we denote

$$
\left[\beta_{0}, \alpha_{0}\right]=\left\{x \in \mathcal{F}: \beta_{0}(t) \leq x(t) \leq \alpha_{0}(t), \quad t \in J\right\},
$$

and we write $\beta_{0} \leq \alpha_{0}$ if $\beta_{0}(t) \leq \alpha_{0}(t)$ for all $t \in J$. 
Theorem 3.1. Let the following conditions hold.

$\left(H_{1}\right)$ The functions $\alpha_{0}$ and $\beta_{0}$ are lower and upper solutions of $B V P(1.1)$, respectively, such that $\beta_{0}(t) \leq \alpha_{0}(t)$ on $J$.

$\left(H_{2}\right)$ The function $f \in C\left(J \times R^{3}, R\right)$ satisfies

$$
f(t, x, y, z)-f(t, \bar{x}, \bar{y}, \bar{z}) \leq M(x-\bar{x})+H(y-\bar{y})+K(z-\bar{z}),
$$

for $\beta_{0}(t) \leq \bar{x}(t) \leq x(t) \leq \alpha_{0}(t),\left(F \beta_{0}\right)(t) \leq \bar{y}(t) \leq y(t) \leq\left(F \alpha_{0}\right)(t)\left(S \beta_{0}\right)(t) \leq \bar{z}(t) \leq z(t) \leq\left(S \alpha_{0}\right)(t) t \in J$.

$\left(H_{3}\right)$ The function $I_{k} \in C(R, R)$ satisfies

$$
I_{k}\left(\sum_{l=1}^{c_{k}} \rho_{l}^{k} x\left(\eta_{l}^{k}\right)\right)-I_{k}\left(\sum_{l=1}^{c_{k}} \rho_{l}^{k} y\left(\eta_{l}^{k}\right)\right) \leq L_{k} \sum_{l=1}^{c_{k}} \rho_{l}^{k}\left(x\left(\eta_{l}^{k}\right)-\gamma\left(\eta_{l}^{k}\right)\right),
$$

whenever $\beta_{0}\left(\eta_{l}^{k}\right) \leq y\left(\eta_{l}^{k}\right) \leq x\left(\eta_{l}^{k}\right) \leq \alpha_{0}\left(\eta_{l}^{k}\right), l=1,2, \ldots, c_{k}, c_{k} \in N=\{1,2, \ldots\}, L_{k} \geq$ $0, k=1,2, \ldots, m$.

$\left(H_{4}\right)$ Inequalities (2.6) and (2.9) hold.

Then there exist monotone sequences $\left\{\alpha_{n}\right\},\left\{\beta_{n}\right\} \subset \mathcal{F}$ such that $\lim _{n \rightarrow \infty} \alpha_{n}(t)=x^{*}(t)$, $\lim _{n \rightarrow \infty} \beta_{n}(t)=x_{*}(t)$ uniformly on $J$ and $x^{*}, x_{*}$ are maximal and minimal solutions of $B V P(1.1)$, respectively, such that

$$
\beta_{0} \leq \beta_{1} \leq \beta_{2} \leq \cdots \leq \beta_{n} \leq x_{*} \leq x \leq x^{*} \leq \alpha_{n} \leq \cdots \leq \alpha_{2} \leq \alpha_{1} \leq \alpha_{0}
$$

on J, where $x$ is any solution of $B V P(1.1)$ such that $\beta_{0}(t) \leq x(t) \leq \alpha_{0}(t)$ on J.

Proof. For any $\sigma \in\left[\beta_{0}, \alpha_{0}\right]$, we consider BVP (2.1) with

$$
v(t)=f(t, \sigma(t),(F \sigma)(t),(S \sigma)(t))-M \sigma(t)-H(F \sigma)(t)-K(S \sigma)(t) .
$$

By Lemma 2.2, BVP (2.1) has a unique solution $x(t)$ for $t \in J$. We define an operator $A$ by $x=A \sigma$, then the operator $A$ is an operator from $\left[\beta_{0}, \alpha_{0}\right]$ to $\mathcal{F}$ and $A$ has the following properties.

(i) $\beta_{0} \leq A \beta_{0}, A \alpha_{0} \leq \alpha_{0}$;

(ii) For any $\sigma_{1}, \sigma_{2} \in\left[\beta_{0}, \alpha_{0}\right], \sigma_{1} \leq \sigma_{2}$ implies $A \sigma_{1} \leq A \sigma_{2}$.

To prove (i), set $\phi=\beta_{0}-\beta_{1}$, where $\beta_{1}=A \beta_{0}$. Then from $\left(H_{1}\right)$ and (2.1) for $t \in \mathcal{J}$, we have

$$
\begin{aligned}
& \varphi^{\prime}(t)= \beta^{\prime}{ }_{0}(t)-\beta^{\prime}{ }_{1}(t), \\
& \geq f\left(t, \beta_{0}(t),\left(F \beta_{0}\right)(t),\left(S \beta_{0}\right)(t)\right)-\left[M \beta_{1}(t)+H\left(F \beta_{1}\right)(t)+K\left(S \beta_{1}\right)(t)\right. \\
&\left.+f\left(t, \beta_{0}(t),\left(F \beta_{0}\right)(t),\left(S \beta_{0}\right)(t)\right)-M \beta_{0}(t)-H\left(F \beta_{0}\right)(t)-K\left(S \beta_{0}\right)(t)\right] \\
&= M \varphi(t)+H(F \varphi)(t)+K(S \varphi)(t), \\
& \Delta \varphi\left(t_{k}\right)=\Delta \beta_{0}\left(t_{k}\right)-\Delta \beta_{1}\left(t_{k}\right) \\
& \geq I_{k}\left(\sum_{l=1}^{c_{k}} \rho_{l}^{k} \beta_{0}\left(\eta_{l}^{k}\right)\right)-\left[L_{k} \sum_{l=1}^{c_{k}} \rho_{l}^{k} \beta_{1}\left(\eta_{l}^{k}\right)+I_{k}\left(\sum_{l=1}^{c_{k}} \rho_{l}^{k} \beta_{0}\left(\eta_{l}^{k}\right)\right)\right. \\
&\left.\quad-L_{k} \sum_{l=1}^{c_{k}} \rho_{l}^{k} \beta_{0}\left(\eta_{l}^{k}\right)\right] \\
&=L_{k} \sum_{l=1}^{c_{k}} \rho_{l}^{k} \varphi\left(\eta_{l}^{k}\right), \quad k=1,2, \ldots, m,
\end{aligned}
$$


and

$$
\begin{aligned}
\varphi(0) & =\beta_{0}(0)-\beta_{1}(0) \\
& \geq \beta_{0}(T)-\mu \sum_{k=1}^{m} \sum_{l=1}^{c_{k}} \tau_{l}^{k} \beta_{0}\left(\eta_{l}^{k}\right)-\beta_{1}(T)+\mu \sum_{k=1}^{m} \sum_{l=1}^{c_{k}} \tau_{l}^{k} \beta_{0}\left(\eta_{l}^{k}\right) \\
& =\varphi(T) .
\end{aligned}
$$

By Lemma 2.3, we get that $\phi(t) \leq 0$ for all $t \in J$, i.e., $\beta_{0} \leq A \beta_{0}$. Similarly, we can prove that $A \alpha_{0} \leq \alpha_{0}$.

To prove (ii), let $u_{1}=A \sigma_{1}, u_{2}=A \sigma_{2}$, where $\sigma_{1} \leq \sigma_{2}$ on $J$ and $\sigma_{1}, \sigma_{2} \in\left[\beta_{0}, \alpha_{0}\right]$. Set $\phi=$ $u_{1}-u_{2}$. Then for $t \in J$ and by $\left(H_{2}\right)$, we obtain

$$
\begin{aligned}
\varphi^{\prime}(t)= & u_{1}^{\prime}(t)-u_{2}^{\prime}(t) \\
= & M u_{1}(t)+H\left(F u_{1}\right)(t)+K\left(S u_{1}\right)(t)+f\left(t, \sigma_{1}(t),\left(F \sigma_{1}\right)(t),\left(S \sigma_{1}\right)(t)\right) \\
& -M \sigma_{1}(t)-H\left(F \sigma_{1}\right)(t)-K\left(S \sigma_{1}\right)(t)-\left(M u_{2}(t)+H\left(F u_{2}\right)(t)+K\left(S u_{2}\right)(t)\right. \\
& \left.+f\left(t, \sigma_{2}(t),\left(F \sigma_{2}\right)(t),\left(S \sigma_{2}\right)(t)\right)-M \sigma_{2}(t)-H\left(F \sigma_{2}\right)(t)-K\left(S \sigma_{2}\right)(t)\right) \\
\geq & M\left(u_{1}(t)-u_{2}(t)\right)+H\left(F\left(u_{1}-u_{2}\right)\right)(t)+K\left(S\left(u_{1}-u_{2}\right)\right)(t), \\
= & M \varphi(t)+H(F \varphi)(t)+K(S \varphi)(t),
\end{aligned}
$$

and by $(H 3)$;

$$
\begin{aligned}
\Delta \varphi\left(t_{k}\right)= & \Delta u_{1}\left(t_{k}\right)-\Delta u_{2}\left(t_{k}\right) \\
= & L_{k} \sum_{l=1}^{c_{k}} \rho_{l}^{k} u_{1}\left(\eta_{l}^{k}\right)+I_{k}\left(\sum_{l=1}^{c_{k}} \rho_{l}^{k} \sigma_{1}\left(\eta_{l}^{k}\right)\right)-L_{k} \sum_{l=1}^{c_{k}} \rho_{l}^{k} \sigma_{1}\left(\eta_{l}^{k}\right)-\left[L_{k} \sum_{l=1}^{c_{k}} \rho_{l}^{k} u_{2}\left(\eta_{l}^{k}\right)\right. \\
& \left.+I_{k}\left(\sum_{l=1}^{c_{k}} \rho_{l}^{k} \sigma_{2}\left(\eta_{l}^{k}\right)\right)-L_{k} \sum_{l=1}^{c_{k}} \rho_{l}^{k} \sigma_{2}\left(\eta_{l}^{k}\right)\right] \\
\geq & L_{k} \sum_{l=1}^{c_{k}} \rho_{l}^{k}\left[u_{1}\left(\eta_{l}^{k}\right)-u_{2}\left(\eta_{l}^{k}\right)\right]=L_{k} \sum_{l=1}^{c_{k}} \rho_{l}^{k} \varphi\left(\eta_{l}^{k}\right), \quad k=1,2, \ldots, m .
\end{aligned}
$$

It is easy to see that

$$
\begin{aligned}
\varphi(0) & =u_{1}(0)-u_{2}(0) \\
& =u_{1}(T)-\mu \sum_{k=1}^{m} \sum_{l=1}^{c_{k}} \tau_{l}^{k} \sigma_{1}\left(\eta_{l}^{k}\right)-u_{2}(T)+\mu \sum_{k=1}^{m} \sum_{l=1}^{c_{k}} \tau_{l}^{k} \sigma_{2}\left(\eta_{l}^{k}\right) \\
& \geq \varphi(T) .
\end{aligned}
$$

Then by using Lemma 2.3, we have $\phi(t) \leq 0$, which implies that $A \sigma_{1} \leq A \sigma_{2}$.

Now, we define the sequences $\left\{\alpha_{n}\right\},\left\{\beta_{n}\right\}$ such that $\alpha_{n+1}=A \alpha_{n}$ and $\beta_{n+1}=A \beta_{n}$. From (i) and (ii) the sequence $\left\{\alpha_{n}\right\},\left\{\beta_{n}\right\}$ satisfy the inequality

$$
\beta_{0} \leq \beta_{1} \leq \cdots \leq \beta_{n} \leq \cdots \leq \alpha_{n} \leq \cdots \leq \alpha_{1} \leq \alpha_{0},
$$

for all $n \in N$. Obviously, each $\alpha_{n}, \beta_{n}(n=1,2, \ldots)$ satisfy

$$
\left\{\begin{array}{l}
\alpha_{n}^{\prime}(t)=M \alpha_{n}(t)+H\left(F \alpha_{n}\right)(t)+K\left(S \alpha_{n}\right)(t)+f\left(t, \alpha_{n-1}(t),\left(F \alpha_{n-1}\right)(t),\left(S \alpha_{n-1}\right)(t)\right) \\
-M \alpha_{n-1}(t)-H\left(F \alpha_{n-1}\right)(t)-K\left(S \alpha_{n-1}\right)(t), \quad t \in J^{-}, \\
\Delta \alpha_{n}\left(t_{k}\right)=L_{k} \sum_{l=1}^{c_{k}} \rho_{l}^{k} \alpha_{n}\left(\eta_{l}^{k}\right)+I_{k}\left(\sum_{l=1}^{c_{k}} \rho_{l}^{k} \alpha_{n-1}\left(\eta_{l}^{k}\right)\right) \\
\quad-L_{k} \sum_{l=1}^{c_{k}} \rho_{l}^{k} \alpha_{n-1}\left(\eta_{l}^{k}\right), \quad k=1,2, \ldots, m, \\
\alpha_{n}(0)+\mu \sum_{k=1}^{m} \sum_{l=1}^{c_{k}} \tau_{l}^{k} \alpha_{n-1}\left(\eta_{l}^{k}\right)=\alpha_{n}(T),
\end{array}\right.
$$


and

$$
\left\{\begin{aligned}
& \beta^{\prime}{ }_{n}(t)= M \beta_{n}(t)+H\left(F \beta_{n}\right)(t)+K\left(S \beta_{n}\right)(t)+f\left(t, \beta_{n-1}(t),\left(F \beta_{n-1}\right)(t),\left(S \beta_{n-1}\right)(t)\right) \\
&-M \beta_{n-1}(t)-H\left(F \beta_{n-1}\right)(t)-K\left(S \beta_{n-1}\right)(t), \quad t \in J^{-}, \\
& \Delta \beta_{n}\left(t_{k}\right)= L_{k} \sum_{l=1}^{c_{k}} \rho_{l}^{k} \beta_{n}\left(\eta_{l}^{k}\right)+I_{k}\left(\sum_{l=1}^{c_{k}} \rho_{l}^{k} \beta_{n-1}\left(\eta_{l}^{k}\right)\right) \\
& \quad-L_{k} \sum_{l=1}^{c_{k}} \rho_{l}^{k} \beta_{n-1}\left(\eta_{l}^{k}\right), \quad k=1,2, \ldots, m, \\
& \beta_{n}(0)+\mu \sum_{k=1}^{m} \sum_{l=1}^{c_{k}} \tau_{l}^{k} \beta_{n-1}\left(\eta_{l}^{k}\right)=\beta_{n}(T) .
\end{aligned}\right.
$$

Therefore, there exist $x_{*}$ and $x^{*}$, such that $\lim _{n \rightarrow \infty} \beta_{n}=x_{*}$ and $\lim _{n \rightarrow \infty} \alpha_{n}=x^{*}$ uniformly on $J$. Clearly, $x_{*}, x^{*}$ are solutions of BVP (1.1).

Finally, we are going to prove that $x_{*}, x^{*}$ are minimal and maximal solutions of BVP (1.1). Assume that $x(t)$ is any solution of BVP (1.1) such that $x \in\left[\beta_{0}, \alpha_{0}\right]$ and that there exists a positive integer $n$ such that $\beta_{n}(t) \leq x(t) \leq \alpha_{n}(t)$ on $J$. Let $\phi=\beta_{n+1}-x$, then for $t \in J$,

$$
\begin{aligned}
\varphi^{\prime}(t)= & \beta_{n+1}^{\prime}(t)-x^{\prime}(t) \\
= & M \beta_{n+1}(t)+H\left(F \beta_{n+1}\right)(t)+K\left(S \beta_{n+1}\right)(t)+f\left(t, \beta_{n}(t),\left(F \beta_{n}\right)(t),\left(S \beta_{n}\right)(t)\right) \\
& -M \beta_{n}(t)-H\left(F \beta_{n}\right)(t)-K\left(S \beta_{n}\right)(t)-f(t, x(t),(F x)(t),(S x)(t)) \\
\geq & M \varphi(t)+H(F \varphi)(t)+K(S \varphi)(t), \\
\Delta \varphi\left(t_{k}\right)= & \Delta \beta_{n+1}\left(t_{k}\right)-\Delta x\left(t_{k}\right) \\
= & L_{k} \sum_{l=1}^{c_{k}} \rho_{l}^{k} \beta_{n+1}\left(\eta_{l}^{k}\right)+I_{k}\left(\sum_{l=1}^{c_{k}} \rho_{l}^{k} \beta_{n}\left(\eta_{l}^{k}\right)\right)-L_{k} \sum_{l=1}^{c_{k}} \rho_{l}^{k} \beta_{n}\left(\eta_{l}^{k}\right)-I_{k}\left(\sum_{l=1}^{c_{k}} \rho_{l}^{k} x\left(\eta_{l}^{k}\right)\right) \\
\geq & L_{k} \sum_{l=1}^{c_{k}} \rho_{l}^{k}\left[\beta_{n+1}\left(\eta_{l}^{k}\right)-x\left(\eta_{l}^{k}\right)\right]=L_{k} \sum_{l=1}^{c_{k}} \rho_{l}^{k} \varphi\left(\eta_{l}^{k}\right), \quad k=1,2, \ldots, m,
\end{aligned}
$$

and

$$
\begin{aligned}
\varphi(0) & =\beta_{n+1}(0)-x(0) \\
& =\beta_{n+1}(T)-\mu \sum_{k=1}^{m} \sum_{l=1}^{c_{k}} \tau_{l}^{k} \beta_{n}\left(\eta_{l}^{k}\right)-x(T)+\mu \sum_{k=1}^{m} \sum_{l=1}^{c_{k}} \tau_{l}^{k} x\left(\eta_{l}^{k}\right) \\
& \geq \varphi(T) .
\end{aligned}
$$

Then by using Lemma 2.3, we have $\phi(t) \leq 0$, which implies that $\beta_{n+1} \leq x$ on J. Similarly we obtain $x \leq \alpha_{n+1}$ on $J$. Since $\beta_{0} \leq x \leq \alpha_{0}$ on $J$, by induction we get $\beta_{n} \leq \times \leq$ $\alpha_{n}$ on $J$ for every $n$. Therefore, $x_{*}(t) \leq x(t) \leq x^{*}(t)$ on $J$ by taking $n \rightarrow \infty$. The proof is complete.

\section{An example}

In this section, in order to illustrate our results, we consider an example.

Example 4.1. Consider the BVP

$$
\left\{\begin{array}{l}
x^{\prime}(t)=t^{3}(1+x(t))+\frac{1}{54} t\left[\int_{0}^{t} t s x(s) d s\right]^{3}+\frac{1}{81} t^{2}\left[\int_{0}^{1} t s x(s) d s\right]^{3}, \quad t \in J=[0,1], \quad t \neq \frac{1}{2}, \\
\Delta x\left(\frac{1}{2}\right)=\frac{1}{4}\left[\frac{1}{5} x\left(\frac{1}{10}\right)+\frac{3}{10} x\left(\frac{1}{5}\right)+\frac{1}{10} x\left(\frac{3}{10}\right)+\frac{1}{5} x\left(\frac{2}{5}\right)+\frac{1}{5} x\left(\frac{1}{2}\right)\right], \quad k=1, \\
x(0)+\frac{1}{5}\left[\frac{1}{5} x\left(\frac{1}{5}\right)+\frac{2}{5} x\left(\frac{3}{10}\right)+\frac{2}{5} x\left(\frac{1}{2}\right)\right]=x(1),
\end{array}\right.
$$


where $k(t, \quad s)=h(t, \quad s)=t s, \quad m=1$, $t_{1}=\frac{1}{2}, c_{1}=5, \rho_{1}^{1}=\frac{1}{5}, \rho_{2}^{1}=\frac{3}{10}, \rho_{3}^{1}=\frac{1}{10}, \rho_{4}^{1}=\frac{1}{5}, \rho_{5}^{1}=\frac{1}{5}, \eta_{1}^{1}=\frac{1}{10}, \eta_{2}^{1}=\frac{1}{5}, \eta_{3}^{1}=\frac{3}{10^{2}}, \eta_{4}^{1}=\frac{2}{5}, \eta_{5}^{1}=\frac{1}{2}, \tau_{1}^{1}=0, \tau_{2}^{1}=\frac{1}{5}, \tau_{3}^{1}=\frac{2}{5}, \tau_{4}^{1}=0, \tau_{5}^{1}=\frac{2}{5}, \mu=\frac{1}{5}$.

Obviously, $\alpha_{0}=0, \beta_{0}=\left\{\begin{array}{l}-5, t \in\left[0, \frac{1}{2}\right] \\ -6, t \in\left(\frac{1}{2}, 1\right]\end{array}\right]$ are lower and upper solutions for (4.1), respectively, and $\beta_{0} \leq \alpha_{0}$.

Let

$$
f(t, x, y, z)=t^{3}(1+x)+\frac{1}{54} t y^{3}+\frac{1}{81} t^{2} z^{3}
$$

Then,

$$
f(t, x, y, z)-f(t, \bar{x}, \bar{y}, \bar{z}) \leq(x-\bar{x})+\frac{1}{2}(y-\bar{y})+\frac{1}{3}(z-\bar{z})
$$

where $\beta_{0}(t) \leq \bar{x}(t) \leq x(t) \leq \alpha_{0}(t),\left(F \beta_{0}\right)(t) \leq \bar{y}(t) \leq \gamma(t) \leq\left(F \alpha_{0}\right)(t),\left(S \beta_{0}\right)(t) \leq \bar{z}(t) \leq z(t) \leq\left(S \alpha_{0}\right)(t), t \in J$. It is easy to see that

$$
I_{1}\left(\sum_{l=1}^{5} \rho_{l}^{1} x\left(\eta_{l}^{1}\right)\right)-I_{1}\left(\sum_{l=1}^{5} \rho_{l}^{1} y\left(\eta_{l}^{1}\right)\right)=\frac{1}{4} \sum_{l=1}^{5} \rho_{l}^{1}\left(x\left(\eta_{l}^{1}\right)-\gamma\left(\eta_{l}^{1}\right)\right),
$$

whenever $\beta_{0}\left(\eta_{l}^{1}\right) \leq \gamma\left(\eta_{l}^{1}\right) \leq x\left(\eta_{l}^{1}\right) \leq \alpha_{0}\left(\eta_{l}^{1}\right), l=1, \ldots, 5$.

Taking $L_{1}=\frac{1}{4}, M=1, H=\frac{1}{2}, K=\frac{1}{3}$, it follows that

$$
\begin{aligned}
& e^{M T}\left[\int_{0}^{T} H \int_{0}^{s} k(s, r) e^{-M(s-r)} d r+K \int_{0}^{T} h(s, r) e^{-M(s-r)} d r d s\right. \\
& \left.\quad+\sum_{k=1}^{m} L_{k} e^{-M t_{k}}\left(\sum_{l=1}^{c_{k}} \rho_{l}^{k} e^{M \eta_{l}^{k}}\right)\right] \\
& =e\left[\int_{0}^{1} \frac{1}{2} \int_{0}^{s} s r e^{-(s-r)} d r+\frac{1}{3} \int_{0}^{1} s r e^{-(s-r)} d r d s\right. \\
& \left.\quad+\frac{1}{4} e^{-\frac{1}{2}}\left(\frac{1}{5} e^{\frac{1}{10}}+\frac{3}{10} e^{\frac{1}{5}}+\frac{1}{10} e^{\frac{3}{10}}+\frac{1}{5} e^{\frac{2}{5}}+\frac{1}{5} e^{\frac{1}{2}}\right)\right] \\
& \approx 0.9287149 \leq 1,
\end{aligned}
$$

and

$$
\begin{aligned}
& \frac{e^{M T}}{e^{M T}-1} \int_{0}^{T}\left[H \int_{0}^{s} k(s, r) d r+K \int_{0}^{T} h(s, r) d r\right] d s+\frac{e^{M T}}{e^{M T}-1} \sum_{k=1}^{m} L_{k}\left(\sum_{l=1}^{c_{k}} \rho_{l}^{k}\right) \\
& =\frac{e}{e-1} \int_{0}^{1}\left[\frac{1}{2} \int_{0}^{s} \operatorname{srdr}+\frac{1}{3} \int_{0}^{1} \operatorname{srdr}\right] d s+\frac{e}{e-1}\left(\frac{1}{4}\right)\left(\frac{1}{5}+\frac{3}{10}+\frac{1}{10}+\frac{1}{5}+\frac{1}{5}\right) \\
& \approx 0.6261991<1 .
\end{aligned}
$$

Therefore, (4.1) satisfies all conditions of Theorem 3.1. So, BVP (4.1) has minimal and maximal solutions in the segment $\left[\beta_{0}, \alpha_{0}\right]$.

\section{Acknowledgements}

This research is supported by the Centre of Excellence in Mathematics, the Commission on Higher Education, 


\section{Author details}

'Department of Mathematics, Faculty of Science, King Mongkut's Institute of Technology Ladkrabang, Bangkok 10520, Thailand 'Department of Mathematics, Faculty of Applied Science, King Mongkut's University of Technology North Bangkok, Bangkok 10800, Thailand ${ }^{3}$ Centre of Excellence in Mathematics, CHE, Sri Ayutthaya Road, Bangkok 10400, Thailand

\section{Authors' contributions}

All authors contributed equally in this article. They read and approved the final manuscript.

\section{Competing interests}

The authors declare that they have no competing interests.

Received: 8 August 2011 Accepted: 5 April 2012 Published: 5 April 2012

\section{References}

1. Lakshmikantham, V, Bainov, DD, Simeonov, PS: Theory of Impulsive Differential Equations. World Scientific, Singapore. (1989)

2. Bainov, DD, Simeonov, PS: Impulsive Differential Equations: Periodic Solutions and Applications. Longman Scientific \& Technical, New York. (1993)

3. Samoilenko, AM, Perestyuk, NA: Impulsive Differential Equations. World Scientific, Singapore. (1995)

4. Ding, $\mathrm{W}, \mathrm{Mi}, \mathrm{J}, \mathrm{Han}, \mathrm{M}$ : Periodic boundary value problems for the first order impulsive functional differential equations. Appl Math Comput. 165, 433-446 (2005). doi:10.1016/j.amc.2004.06.022

5. Zhang, F, Li, M, Yan, J: Nonhomogeneous boundary value problem for first-order impulsive differential equations with delay. Comput Math Appl. 51, 927-936 (2006). doi:10.1016/j.camwa.2005.11.028

6. Chen, L, Sun, J: Nonlinear boundary problem of first order impulsive integro-differential equations. J Comput Appl Math. 202, 392-401 (2007). doi:10.1016/j.cam.2005.10.041

7. Liang, R, Shen, J: Periodic boundary value problem for the first order impulsive functional differential equations. J Comput Appl Math. 202, 498-510 (2007). doi:10.1016/j.cam.2006.03.017

8. Ding, W, Xing, Y, Han, M: Anti-periodic boundary value problems for first order impulsive functional differential equations. Appl Math Comput. 186, 45-53 (2007). doi:10.1016/j.amc.2006.07.087

9. Yang, $X$, Shen, J: Nonlinear boundary value problems for first order impulsive functional differential equations. Appl Math Comput. 189, 1943-1952 (2007). doi:10.1016/j.amc.2006.12.085

10. Luo, Z, Jing, Z: Periodic boundary value problem for first-order impulsive functional differential equations. Comput Math Appl. 55, 2094-2107 (2008). doi:10.1016/j.camwa.2007.08.036

11. Wang, X, Zhang, J: Impulsive anti-periodic boundary value problem of first-order integro-differential equations. J Comput Appl Math. 234, 3261-3267 (2010). doi:10.1016/j.cam.2010.04.024

12. Song, $G$, Zhao, $Y$, Sun, $X:$ Integral boundary value problems for first order impulsive integro-differential equations of mixed type. J Comput Appl Math. 235, 2928-2935 (2011). doi:10.1016/j.cam.2010.12.007

13. Nieto, JJ, Rodríguez-López, R: Existence and approximation of solutions for nonlinear functional differential equations with periodic boundary value conditions. Comput Appl Math. 40, 433-442 (2000). doi:10.1016/S0898-1221(00)00171-1

14. Nieto, JJ, Rodríguez-López, R: Periodic boundary value problem for non-Lipschitzian impulsive functional differential equations. J Math Anal Appl. 318, 593-610 (2006). doi:10.1016/j.jmaa.2005.06.014

15. He, Z, Yu, J: Periodic boundary value problem for first-order impulsive functional differential equations. J Comput Appl Math. 138, 205-217 (2002). doi:10.1016/50377-0427(01)00381-8

16. He, Z, Yu, J: Periodic boundary value problem for first-order impulsive ordinary differential equations. J Math Anal Appl. 272, 67-78 (2002). doi:10.1016/S0022-247X(02)00133-6

17. Chen, L, Sun, J: Nonlinear boundary value problem of first order impulsive functional differential equations. J Math Anal Appl. 318, 726-741 (2006). doi:10.1016/j.jmaa.2005.08.012

18. Chen, L, Sun, J: Nonlinear boundary value problem for first order impulsive integro-differential equations of mixed type. J Math Anal Appl. 325, 830-842 (2007). doi:10.1016/j.jmaa.2006.01.084

19. Wang, G, Zhang, L, Song, G: Extremal solutions for the first order impulsive functional differential equations with upper and lower solutions in reversed order. J Comput Appl Math. 235, 325-333 (2010). doi:10.1016/..cam.2010.06.014

20. Zhang, L: Boundary value problem for first order impulsive functional integro-differential equations. J Comput Appl Math. 235, 2442-2450 (2011). doi:10.1016/j.cam.2010.10.045

21. Zhang, Y, Zhang, F: Multi-point boundary value problem of first order impulsive functional differential equations. J Appl Math Comput. 31, 267-278 (2009). doi:10.1007/s12190-008-0209-2

22. Tariboon, J: Boundary value problems for first order functional differential equations with impulsive integral conditions. J Comput Appl Math. 234, 2411-2419 (2010). doi:10.1016/j.cam.2010.03.007

23. Liu, Z, Han, J, Fang, L: Integral boundary value problems for first order integro-differential equations with impulsive integral conditions. Comput Math Appl. 61, 3035-3043 (2011). doi:10.1016/j.camwa.2011.03.094

doi:10.1186/1687-2770-2012-38

Cite this article as: Thaiprayoon et al:: Multi-point boundary value problem for first order impulsive integro-

differential equations with multi-point jump conditions. Boundary Value Problems 2012 2012:38. 